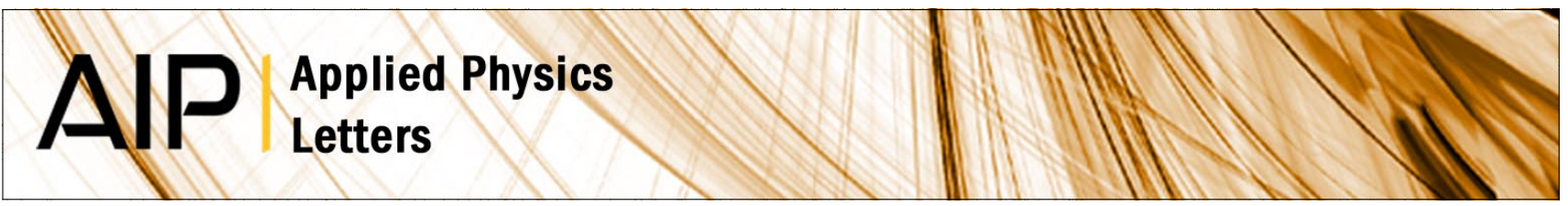

\title{
Effect of pre-tension on the peeling behavior of a bio-inspired nano-film and a hierarchical adhesive structure
}

Zhilong Peng and Shaohua Chen

Citation: Appl. Phys. Lett. 101, 163702 (2012); doi: 10.1063/1.4758481

View online: http://dx.doi.org/10.1063/1.4758481

View Table of Contents: http://apl.aip.org/resource/1/APPLAB/v101/i16

Published by the American Institute of Physics.

\section{Related Articles}

The sensitivity of graphene "snap-through" to substrate geometry Appl. Phys. Lett. 100, 233111 (2012)

Thin smart multilayer microwave absorber based on hybrid structure of polymer and carbon nanotubes Appl. Phys. Lett. 100, 213105 (2012)

An off-lattice, self-learning kinetic Monte Carlo method using local environments

J. Chem. Phys. 135, 174103 (2011)

An efficient algorithm to accelerate the discovery of complex material formulations

J. Chem. Phys. 132, 174103 (2010)

Structural colors from Morpho peleides butterfly wing scales

J. Appl. Phys. 106, 074702 (2009)

\section{Additional information on Appl. Phys. Lett.}

Journal Homepage: http://apl.aip.org/

Journal Information: http://apl.aip.org/about/about_the_journal

Top downloads: http://apl.aip.org/features/most_downloaded

Information for Authors: http://apl.aip.org/authors

\section{ADVERTISEMENT}

\section{AIP Applied Physics Letters}

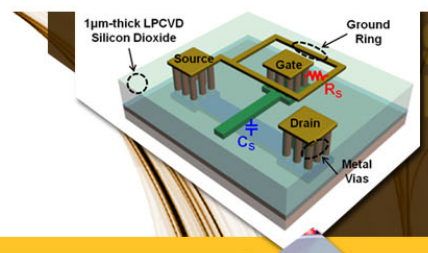

\section{SURFACES AND} INTERFACES

Focusing on physical, chemical, biological structural, optical, magnetic and electrical properties of surfaces and interfaces, and more..

\section{EXPLORE WHAT'S NEW IN APL}

SUBMIT YOUR PAPER NOW!
ENERCY CONVERSION AND STORACE 


\title{
Effect of pre-tension on the peeling behavior of a bio-inspired nano-film and a hierarchical adhesive structure
}

\author{
Zhilong Peng and Shaohua Chen ${ }^{\text {a) }}$ \\ LNM, Institute of Mechanics, Chinese Academy of Sciences, Beijing 100190, China
}

(Received 15 August 2012; accepted 27 September 2012; published online 15 October 2012)

\begin{abstract}
Inspired by the reversible adhesion behaviors of geckos, the effects of pre-tension in a bio-inspired nano-film and a hierarchical structure on adhesion are studied theoretically. In the case with a uniformly distributing pre-tension in a spatula-like nano-film under peeling, a closed-form solution to a critical peeling angle is derived, below or above which the peel-off force is enhanced or reduced, respectively, compared with the case without pre-tension. The effects of a non-uniformly distributing pre-tension on adhesion are further investigated for both a spatula-like nano-film and a hierarchical structure-like gecko's seta. Compared with the case without pre-tension, the pre-tension, no matter uniform or non-uniform, can increase the adhesion force not only for the spatula-like nano-film but also for the hierarchical structure at a small peeling angle, while decrease it at a relatively large peeling angle. Furthermore, if the pre-tension is large enough, the effective adhesion energy of a hierarchical structure tends to vanish at a critical peeling angle, which results in spontaneous detachment of the hierarchical structure from the substrate. The present theoretical predictions can not only give some explanations on the existing experimental observation that gecko's seta always detaches at a specific angle and no apparent adhesion force can be detected above the critical angle but also provide a deep understanding for the reversible adhesion mechanism of geckos and be helpful to the design of biomimetic reversible adhesives. (C) 2012 American Institute of Physics. [http://dx.doi.org/10.1063/1.4758481]
\end{abstract}

The exceptional adhesion ability of geckos to climb and detach from almost any surfaces at will has been attracting many scientists' interests since Aristotle who observed geckos to "run up and down a tree in any way, even with the head downwards" in the 4th century B.c. ${ }^{1}$ The underlying mechanisms of geckos' attachment and detachment, including suction, secretions, interlocking and so on, have been proposed and then rejected in the past decades. Until recently, the micro-structures of geckos' adhesive system were observed and the fundamental principle of geckos' adhesion was disclosed with the help of the advanced experimental methods. ${ }^{2-4}$ It is found that one toe of gecko's foot consists of hundreds of thousands of setae and each seta further branches into hundreds of spatulae. The dominant mechanism of gecko's adhesion is due to the van der Waals force, ${ }^{1,3}$ while capillary force might also play a significant role. $^{5-7}$ Using such a hierarchical structure, micro-scale forces, such as van der Waals force and capillary one, are accumulated to support geckos' body weight. Furthermore, it is widely believed that geckos rely on the hierarchical adhesive structure to achieve the robust attachment and easy detachment.

Many interesting works have been extensively done in order to investigate the micro-scale mechanism of geckos' adhesion. ${ }^{8-14}$ Gao et al. ${ }^{9}$ assumed a spatula on gecko's foot as a cylindrical fiber, and found that the interfacial adhesion strength could achieve the theoretical one only if the radius of the fiber was below a critical size. Considering the real profile of a spatula that looks like a nano-film with a finite

\footnotetext{
${ }^{\text {a) }}$ Author to whom correspondence should be addressed. Electronic mail: chenshaohua72@hotmail.com. Tel.: 86-10-82543960.
}

length scale, the classical Kendall's model was often adopted to simulate the peeling behavior of geckos' spatulae. ${ }^{15-17}$ Tian et al. ${ }^{10}$ proposed a frictional adhesion model and found the peel-off force varying significantly with the peeling angle. The adhesive behavior of a bio-inspired nano-film with a finite contact length was investigated by Peng et al., ${ }^{12}$ in which the effect of the adhesive length on adhesion force was considered. The influences of surface roughness ${ }^{18}$ and environmental relative humidity ${ }^{6}$ on nano-film's adhesion were further studied. All the above theoretical results are helpful to explain some existing experimental observations of geckos' adhesion, such as the size effect of a spatula and the rolling behavior of geckos' feet for reversible adhesion.

Another important experimental finding is that gecko's foot always slides a small distance when it adheres to a solid surface. ${ }^{3}$ The sliding behavior helps geckos to achieve larger intimate contact area on a rough surface. Simultaneously, pre-tension will be induced in the nano-film-like spatula due to the sliding. Chen et al. ${ }^{17}$ investigated the effect of pretension on the peel-off force using an elastic tape model, in which pre-tension was assumed to distribute uniformly in the tape. The results showed that pre-tension could significantly increase the peel-off force at a small peeling angle, while decrease it at a large one. However, the critical peeling angle below and above which the peel-off force is increased and decreased was not given in their study. Furthermore, according to the sliding behavior of geckos' feet, pre-tension generated in the spatular pad should be significantly non-uniform. Numerical calculation has proved a linearly distributing pretension in the spatular pad due to the sliding behavior. ${ }^{19}$ How the non-uniform pre-tension affects the adhesion of the spatular pad is still an open question. 
In addition, it is well known that biology often resorts to a hierarchical adhesive system to achieve robust attachment and easy detachment. The mechanical mechanism of such a hierarchical structure has been investigated by several theoretical models, such as a hierarchical fibrillar model ${ }^{20}$ and a three-level hierarchical spring model. ${ }^{21}$ Specially, a relatively real hierarchical model was proposed by Chen et al., ${ }^{16}$ in which the intermediate level of the structure consists of setae, while the lowest level was modeled as spatulae. However, the above hierarchical models could not account for an important experimental observation ${ }^{22}$ that a critical detachment angle independent on the peeling force existed for gecko's hierarchical setae and even for the entire toe. Above the critical angle, no apparent adhesion force could be detected for the hierarchical structure. What factor leads to such an interesting phenomenon? Is it also due to the pretension in the lowest level of the hierarchical system?

In this letter, in order to answer the above questions, the effect of a uniform pre-tension on the adhesion of a spatulalike nano-film is further investigated first based on Ref. 17. Then, considering the effect of a non-uniform pre-tension, theoretical models of a spatula-like nano-film and a twolevel hierarchical structure are established and investigated. Finally, the results are further extended to a higher level hierarchical system.

Considering the case with a uniformly distributing pretension in a spatula-like nano-film as shown in Fig. 1, Chen et al. ${ }^{17}$ found that the pre-tension could significantly increase the peel-off force at a small peeling angle, while decrease it at a large one, leading to a strongly reversible adhesion. Based on the analysis of Chen et al., ${ }^{17}$ a critical peeling angle $\theta_{0}$ can be further obtained, which depends on the value of the pre-tension. Below or above the critical angle, the peel-off force can be enhanced or reduced compared with the case without pre-tension.

According to the classical Kendall's peeling model, the peel-off force $P$ of an elastic nano-film without pre-tension as a function of the peeling angle $\theta$ can be expressed as ${ }^{15}$

$$
P=\frac{2 \Delta \gamma}{\sqrt{(1-\cos \theta)^{2}+2 \Delta \gamma / E h}+(1-\cos \theta)} .
$$

If the nano-film is initially subjected to a pre-tension $P_{0}$, the peel-off force can be written as, ${ }^{17}$

$$
\begin{aligned}
P= & E h\left[\sqrt{\left(1-\cos \theta-P_{0} / E h\right)^{2}-\left(P_{0} / E h\right)^{2}+2 \Delta \gamma / E h}\right. \\
& \left.-\left(1-\cos \theta-P_{0} / E h\right)\right]
\end{aligned}
$$

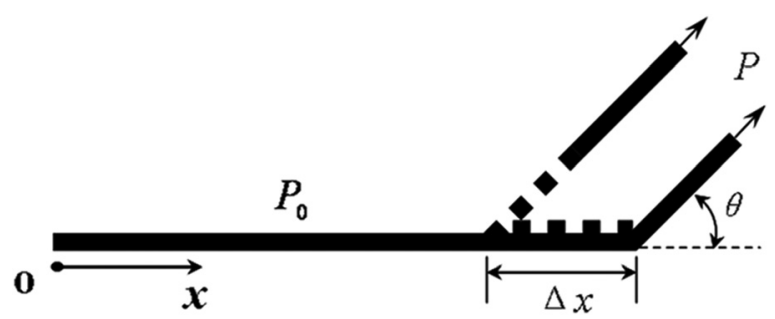

FIG. 1. Schematic of a spatula-like nano-film with pre-tension $P_{0}$ attached on a rigid substrate. $P_{0}$ can be either a constant or a function of the coordinate $x . P$ is a peeling force and $\theta$ is the peeling angle. $\Delta x$ denotes an infinitesimal peeling distance of the nano-film. where $\Delta \gamma$ is the interfacial adhesion energy, $h$ is the film thickness, and $E$ the Young's modulus of the film.

Letting Eq. (1) equal Eq. (2) yields a critical peeling angle $\theta_{0}$ as a function of the pre-tension $P_{0}$,

$$
\theta_{0}=\cos ^{-1}\left(1-\frac{2 \Delta \gamma}{P_{0}}+\frac{P_{0}}{4 E h}\right) .
$$

In contrast to the peel-off force of the model without pretension, if the peeling angle is above the critical one, the peel-off force of the nano-film with a determined pre-tension will be decreased; while the peeling angle is below the critical one, the peel-off force will be increased. That means the pretension will increase the interfacial adhesion strength at a peeling angle that is smaller than the critical one, while it will reduce the interfacial adhesion strength at a peeling angle that is larger than the critical one. In one word, robust attachment can be achieved at a lower peeling angle and easy detachment will be realized at a larger one via the effect of pre-tension.

The critical peeling angle $\theta_{0}$ as a function of the pretension $P_{0}$ (the attachment and detachment map of a spatula) is shown in Fig. 2 with the parameter $\Delta \gamma / E h=1 \times 10^{-3}$. One can see that the critical peeling angle decreases quickly when the pre-tension increases. When $P_{0}$ vanishes, the critical angle does not exist, which corresponds to the model without pre-tension.

In order to ensure stable adhesion and avoid spontaneous detachment of the nano-film with a uniform pre-tension from a substrate, the pre-tension should satisfy $P_{0}<\sqrt{2 E h \Delta \gamma}$, which can be obtained from the competition between the adhesion energy and the strain energy induced by the pre-tension.

Naturally, geckos slide their feet a small distance on a solid surface before achieving robust adhesion, ${ }^{3}$ the pretension resulting from which is not necessarily uniform as investigated in Ref. 17. The distribution of pre-tension in the spatula depends on the interfacial friction as shown in Fig. 1, where the pre-tension $P_{0}$ is not a constant but a function of variable $x$.

Two distributing states of the non-uniform pre-tension in the nano-film are possible: (a) when the dragging force is

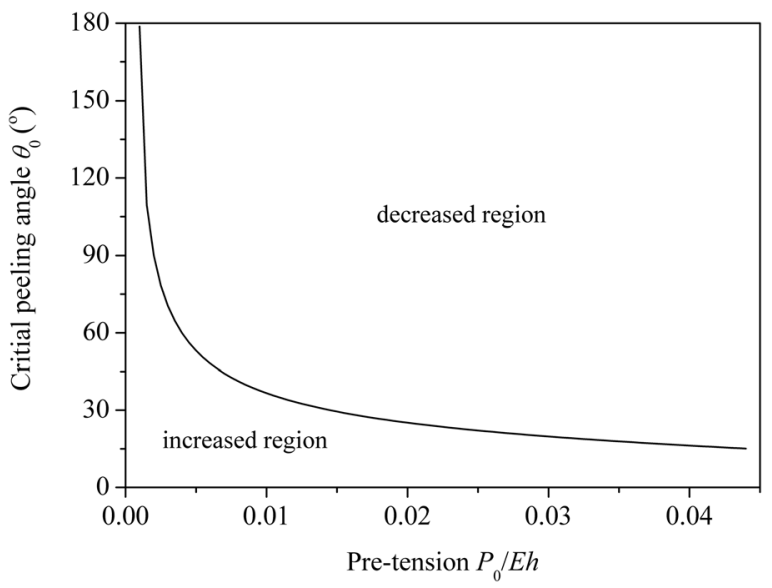

FIG. 2. The critical peeling angle $\theta_{0}$ as a function of the non-dimensional pre-tension $P_{0} / E h$ in the spatula-like nano-film. Two regions are divided by the relationship curve, below which the adhesion force is improved and above which the adhesion force is reduced in contrast to the case without the pre-tension. 
smaller than the maximum static frictional force of the interface, the spatula-like nano-film remains sticking on the solid surface. We assume a linearly distributing interface frictional traction $\tau=k x$, where $k$ is a constant. The pre-tension distribution in the spatula-like nano-film can be found according to $P_{0}(x)=1 / 2 k x^{2}$; (b) when the dragging force is larger than the maximum static frictional force, the spatula-like nano-film will slide a small distance and then adhere on the solid surface. We assume a uniformly distributing frictional traction equaling the interfacial shear strength $\tau=\tau_{0}$, which results in the pre-tension distribution in the nano-film $P_{0}(x)=\tau_{0} x$. For other possible distributing forms of the interface frictional traction, the similar method can be used to find the pre-tension in the nano-film.

Similar to the classical Kendall's work, ${ }^{15}$ we assume a steady-state virtually infinitesimal peeling length $\Delta x$ as shown in Fig. 1. The work done by the applied peeling force is

$$
W_{1}=P \Delta x(1-\cos \theta)+P \Delta x \frac{P-P_{0}}{E h},
$$

where the pre-tension $P_{0}$ is a function of variable $x, \Delta x(1$ $-\cos \theta)$ corresponds to the distance in the peeling direction, and $\Delta x\left[\left(P-P_{0}\right) / E h\right]$ from the additional elastic deformation of $\Delta x$.

Summation of the adhesion energy and the elastic energy stored in the film is

$$
W_{2}=\Delta \gamma \cdot \Delta x+\frac{1}{2} \frac{\Delta x}{E h}\left(P^{2}-P_{0}^{2}\right) .
$$

Then, the energy conservation leads to

$$
\frac{P^{2}}{2 E h}+P(1-\cos \theta)-\frac{P P_{0}}{E h}+\frac{P_{0}^{2}}{2 E h}-\Delta \gamma=0
$$

from which one can see the peel-off force $P$ depends not only on the pre-tension $P_{0}$ but also on the peeling angle $\theta$.

Figure 3 gives the peel-off force along the film's detached length at several peeling angles for the sticking case $P_{0}(x)=1 / 2 k x^{2}$ and the sliding case $P_{0}(x)=\tau_{0} x$ with nondimensional parameters $k h / E=3.75 \times 10^{-5}, L / h=40$, and $\tau_{0} / E=10^{-3}$, in which the case without pre-tension is also

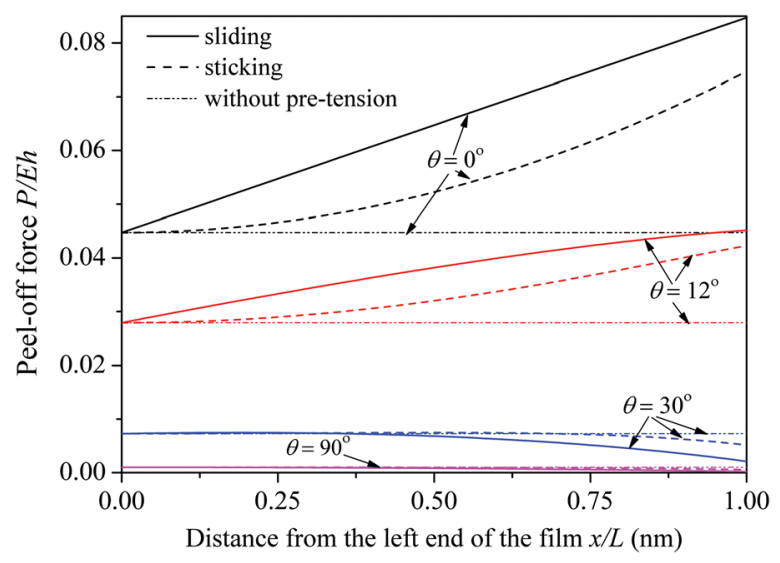

FIG. 3. The non-dimensional steady-state peel-off force $P / E h$ varying with the detached length for cases with different pre-tension distributing forms and different peeling angles. The results for the case without pre-tension are also given for comparison. given for comparison. It is shown that the initial peel-off forces at $x / L=1.0$ at small peeling angles for both the sliding and the sticking cases, e.g., $\theta=0^{\circ}, 12^{\circ}$, are much larger than that in the corresponding cases without pre-tension. All the steady-state peel-off forces along the peeling length in the pre-tension cases with small peeling angles are improved in contrast to those in the corresponding cases without pretension. However, the initial peel-off forces at $x / L=1.0$ at relatively large peeling angles for both the sliding and the sticking cases, e.g., $\theta>30^{\circ}$, are much smaller than that in the corresponding cases without the pre-tension. All the steady-state peel-off forces along the peeling length in the pre-tension cases with relatively large peeling angles are also reduced in contrast to those in the corresponding cases without pre-tension. All the results in Fig. 3 show that pretension, even distributing non-uniformly in the nano-film, always improves the adhesion force of the nano-film at a small peeling angle, while reduces the adhesion force at a relatively large one. In a word, pre-tension, no matter distributing uniformly or non-uniformly in the spatula-like nano-film, helps geckos to achieve robust adhesion at a small peeling angle and easy detachment at a relatively large one.

In addition, it is known that the adhesive system of gecko consists of hundreds of thousands of setae and each seta branches into hundreds of spatulae, which typically belongs to be a hierarchical structure. It was experimentally found that a single seta or seta array could detach from a substrate at a critical angle with hardly any adhesion force. ${ }^{22}$ What is the reason? Inspired by this question, a hierarchical model similar to that in Ref. 16 is established in the present paper as shown in Fig. 4, which consists of two levels, i.e., seta and spatulae. Furthermore, the pre-tension in the first level (spatula) is included in our model, whose effect will be investigated on the adhesion of the two-level hierarchical structure.

Without considering pre-tension, the effective adhesion energy of the second-level of the hierarchical structure, i.e., a single seta, consists of the energy dissipated along the interface and the elastic strain energy stored in the spatulae, which can be expressed as ${ }^{16}$

$$
\Delta \gamma_{2}=\varphi_{1}\left(\Delta \gamma+\frac{P^{2} L}{2 E h^{2}}\right)
$$

where $\varphi_{1}$ is the area fraction of spatulae at the end of a seta, $\Delta \gamma$ is the interfacial adhesion energy between a spatula and a substrate, $L$ is the shaft length of a spatula, and $h$ the thickness

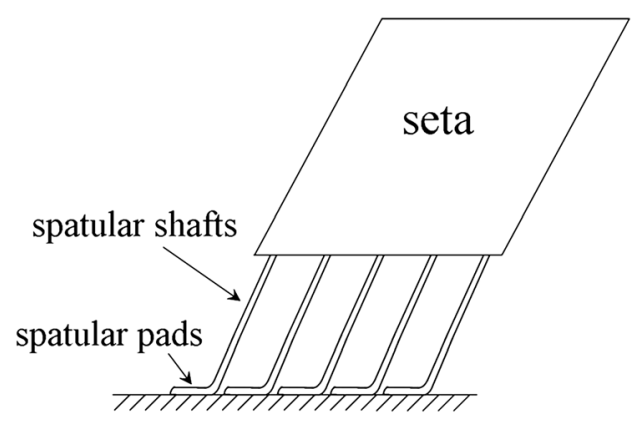

FIG. 4. Schematic of a two-level hierarchical adhesive structure, which consists of several spatulae (the first level) and a seta (the second-level). 
of a spatula. Here, one should be noted that $P$ in Eq. (7) denotes the peeling force exerted at the end of each spatula.

With pre-tension in the first level (spatula), the effective adhesion energy of the second-level (seta) can be rewritten as

$$
\Delta \gamma_{2}=\varphi_{1}\left[\Delta \gamma+\frac{L}{2 E h^{2}}\left(P^{2}-P_{0}^{2}\right)\right]
$$

where $P_{0}$ is the pre-tension in each spatula. From Eq. (8), one can see that the pre-tension in the first level will show obvious influence on the effective adhesion energy of the second level. Equation (8) can be further expressed as a nondimensional form

$$
\frac{\Delta \gamma_{2}}{\Delta \gamma}=\varphi_{1}\left\{1+\frac{E L}{2 \Delta \gamma}\left[\left(\frac{P}{E h}\right)^{2}-\left(\frac{P_{0}}{E h}\right)^{2}\right]\right\} .
$$

The effective adhesion energy of the second level is plotted as a function of the peeling angle with $\varphi_{1}=0.6$, $E L / \Delta \gamma=1.6 \times 10^{5}$, and different values of pre-tension in Fig. 5, where the result without considering pre-tension is also given for comparison. It is shown that, for a determined pre-tension, the effective adhesion energy of the second level is improved at small peeling angles, while it is reduced at relatively large ones. As discussed in one of our previous works, ${ }^{18}$ the adhesion force of a structure is almost linearly proportional to the effective adhesion energy. Therefore, pre-tension in the first level also improves the adhesion force of the hierarchical structure at small peeling angles, while reduces it at large ones as shown in Fig. 5.

Similar to the effect of pre-tension on the adhesion of a spatula-like nano-film, a critical peeling angle $\theta_{\mathrm{cr} 1}$ for the second level of the hierarchical structure can also be obtained by equating Eqs. (7) and (8),

$$
\begin{aligned}
& {\left[\sqrt{\left(1-\cos \theta_{\mathrm{cr} 1}-P_{0} / E h\right)^{2}-\left(P_{0} / E h\right)^{2}+2 \Delta \gamma / E h}\right.} \\
& \left.\quad-\left(1-\cos \theta_{\mathrm{cr} 1}-P_{0} / E h\right)\right]^{2} \\
& =\left[\sqrt{\left(1-\cos \theta_{\mathrm{cr} 1}\right)^{2}+2 \Delta \gamma / E h}-\left(1-\cos \theta_{\mathrm{cr} 1}\right)\right]^{2}+\left(\frac{P_{0}}{E h}\right)^{2}
\end{aligned}
$$

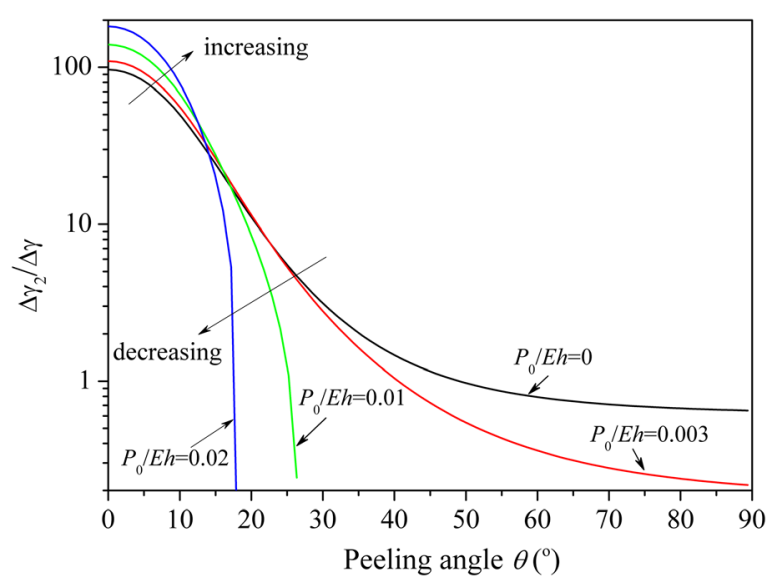

FIG. 5. The effective adhesion energy of the two-level hierarchical adhesive structure as a function of the peeling angle for cases with different pretensions in the first level. below or above which the pre-tension will increase or decrease the adhesion force of the second level of the hierarchical structure, respectively.

From Fig. 5, one may note that the effective adhesion energy of the second level will vanish at another critical peeling angle when the pre-tension is large enough. Then, the relationship $\Delta \gamma_{2}=0$ leads to another critical peeling angle $\theta_{\mathrm{cr} 2}$ of the second level as a function of the pre-tension,

$$
\begin{aligned}
& {\left[\sqrt{\left(1-\cos \theta_{\mathrm{cr} 2}-P_{0} / E h\right)^{2}-\left(P_{0} / E h\right)^{2}+2 \Delta \gamma / E h}\right.} \\
& \left.-\left(1-\cos \theta_{\mathrm{cr} 2}-P_{0} / E h\right)\right]^{2} \\
& =\left(\frac{P_{0}}{E h}\right)^{2}-\frac{2 \Delta \gamma}{E L},
\end{aligned}
$$

which means a spontaneous detachment of the hierarchical structure from the substrate at peeling angles that are larger than $\theta_{\mathrm{cr} 2}$ because of the vanishing effective adhesion energy.

Figure 6 gives the two critical angles $\theta_{\text {cr1 }}$ and $\theta_{\text {cr2 }}$ as a function of the pre-tension (also the attachment and detachment maps of a hierarchical structure), in which the material parameters are adopted as those of a gecko's seta. From Fig. 6, one can infer that the effective adhesion energy of the hierarchical structure vanishes at $\theta=30^{\circ}$ when the nondimensional pre-tension $P_{0} / E h$ equals about 0.008 . This result can effectively explain the experimental observation that geckos' setae always tend to detach at a peeling angle approximately $30^{\circ}$, and no apparent adhesion force is detected at this moment. ${ }^{22}$ That is to say geckos can generate an appropriate pre-tension in their spatulae that leads to a spontaneous detachment of the hierarchical adhesive system at a critical peeling angle, which is very important for their easy detachment from substrates when they prey on small animals or escape from the predators.

For a three-level hierarchical structure, and even more levels' hierarchical one, a method similar to Ref. 20 can be adopted to find the effective adhesion energy at each level,

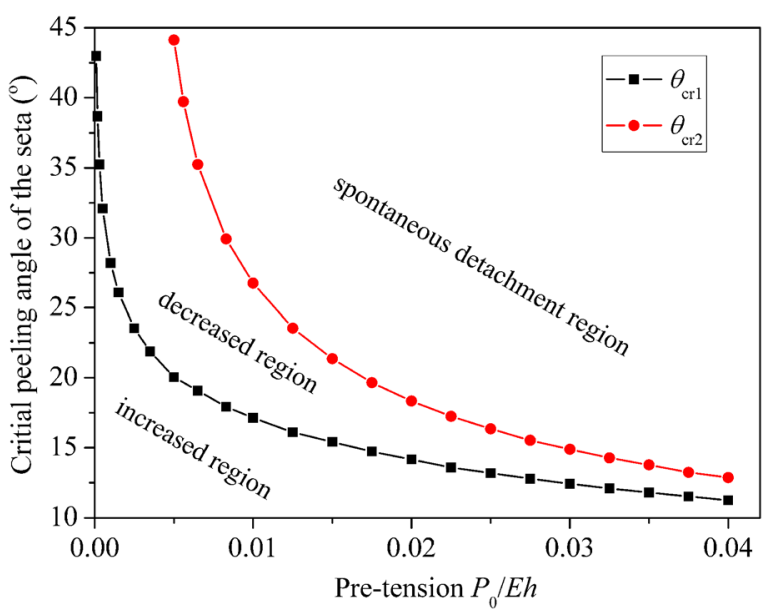

FIG. 6. Two critical peeling angles of the two-level hierarchical adhesive structure varying with the pre-tension. $\theta_{\mathrm{cr} 1}$ denotes the critical angle, below which the adhesion force of the second-level of the hierarchical structure will be improved and above which the adhesion force will be reduced. $\theta_{\mathrm{cr} 2}$ is the critical angle, above which the two-level hierarchical adhesive structure will detach from the substrate spontaneously. 
which will be very useful for the bottom-up design of a hierarchical structure. Considering the pre-tension in the firstlevel, the effective adhesion energy of the third level can be written as

$$
\Delta \gamma_{3}=\varphi_{2}\left(\Delta \gamma_{2}+\frac{\left(\sigma_{2}\right)^{2} L_{2}}{2 E}\right)
$$

where $\varphi_{2}, \Delta \gamma_{2}, L_{2}$, and $\sigma_{2}$ are the area fraction, the effective adhesion energy, the fiber length, and the tensile stress in fibers of the second level, respectively. $\sigma_{2}=\varphi_{1} P / h$ and $P$ is the peeling force of the first level in Eq. (2), which is applicable not only to the case with a uniform pre-tension $P_{0}$ but also to the case with a non-uniform one $P_{0}(x)$.

For a higher-level hierarchical structure, an iterative procedure can be used to find the effective adhesion energy at each level. The effective adhesion energy of the $n$th level of a hierarchical structure can be written as

$$
\Delta \gamma_{n}=\varphi_{n-1}\left(\Delta \gamma_{n-1}+\frac{\left(\sigma_{n-1}\right)^{2} L_{n-1}}{2 E}\right)
$$

where $\sigma_{n-1}=\varphi_{n-2} \sigma_{n-2}=\varphi_{n-2} \varphi_{n-3} \sigma_{n-3}=\varphi_{n-2} \varphi_{n-3} \cdots \varphi_{2} \sigma_{2}$ $=\prod_{i=1}^{n-2} \varphi_{i} P / h$.

In conclusion, the effects of pre-tension on the adhesion of a spatula-like nano-film and a hierarchical adhesive structure are investigated in the present paper. Two kinds of distributing forms of the pre-tension are considered: uniform and non-uniform distributions. In the case with a uniformly distributing pre-tension, a closed-form solution to a critical peeling angle is given, below or above which the pre-tension will increase or decrease the adhesion force in contrast to the case without pre-tension. If the pre-tension in the nano-film distributes non-uniformly, we found that the pre-tension, even distributing non-uniformly, can significantly improve the adhesion force at small peeling angles, while decrease it at relatively large ones. The effect of pre-tension on the adhesion feature of a hierarchical adhesive structure is further studied. It is found that pre-tension in the first-level of a hierarchical adhesive structure can enhance not only the adhesion force of the second-level but also that of a higher-level structure at small peeling angles, while decrease them at relatively large ones. Furthermore, spontaneous detachment of the hierarchical structure at a critical peeling angle will happen if the pre-tension in the spatula-like nano-film is large enough. It can be inferred that the pre-tension generated during the geckos' climbing process is very important to achieve robust attachment at small peeling angles and easy detachment at relatively large ones. All the results in the present paper should be useful for the understanding of geckos' reversible adhesive behaviors.

The work reported here was supported by NSFC through Grant Nos. 10972220, \#11125211, \#11021262 and the 973 Nano-project (2012CB937500).

${ }^{1}$ K. Autumn, M. Sitti, Y. C. A. Liang, A. M. Peattie, W. R. Hansen, S. Sponberg, T. W. Kenny, R. Fearing, J. N. Israelachvili, and R. J. Full, Proc. Natl. Acad. Sci. U.S.A. 99, 12252 (2002).

${ }^{2}$ A. P. Russell, A. M. Bauer, and R. Laroiya, J. Zool. 241, 767 (1997).

${ }^{3}$ K. Autumn, Y. A. Liang, S. T. Hsieh, W. Zesch, W. P. Chan, T. W. Kenny, R. Fearing, and R. J. Full, Nature 405, 681 (2000).

${ }^{4}$ G. Huber, S. N. Gorb, R. Spolenak, and E. Arzt, Biol. Lett. 1, 2 (2005).

${ }^{5}$ G. Huber, H. Mantz, R. Spolenak, K. Mecke, K. Jacobs, S. N. Gorb, and E. Arzt, Proc. Natl. Acad. Sci. U.S.A. 102, 16293 (2005).

${ }^{6}$ Z. L. Peng and S. H. Chen, Colloids Surf., B 88, 717 (2011).

${ }^{7}$ W. X. Sun, P. Neuzil, T. S. Kustandi, S. Oh, and V. D. Samper, Biophys. J. 89, L14 (2005).

${ }^{8}$ H. E. Jeong, J. K. Lee, M. K. Kwak, S. H. Moon, and K. Y. Suh, Appl. Phys. Lett. 96, 043704 (2010).

${ }^{9}$ H. J. Gao, X. Wang, H. M. Yao, S. Gorb, and E. Arzt, Mech. Mater. 37, 275 (2005).

${ }^{10}$ Y. Tian, N. Pesika, H. B. Zeng, K. Rosenberg, B. X. Zhao, P. McGuiggan, K. Autumn, and J. Israelachvili, Proc. Natl. Acad. Sci. U.S.A. 103, 19320 (2006).

${ }^{11}$ B. N. J. Persson and S. Gorb, J. Chem. Phys. 119, 11437 (2003).

${ }^{12}$ Z. L. Peng, S. H. Chen, and A. K. Soh, Int. J. Solids Struct. 47, 1952 (2010).

${ }^{13}$ N. S. Pesika, Y. Tian, B. X. Zhao, K. Rosenberg, H. B. Zeng, P. McGuiggan, K. Autumn, and J. N. Israelachvili, J. Adhes. 83, 383 (2007).

${ }^{14}$ J. H. Lee, R. S. Fearing, and K. Komvopoulos, Appl. Phys. Lett. 93, 191910 (2008).

${ }^{15}$ K. Kendall, J. Phys. D: Appl. Phys. 8, 1449 (1975).

${ }^{16}$ B. Chen, P. D. Wu, and H. Gao, Proc. R. Soc. London, Ser. A 464, 1639 (2008).

${ }^{17}$ B. Chen, P. D. Wu, and H. J. Gao, J. R. Soc. Interface 6, 529 (2009).

${ }^{18}$ Z. L. Peng and S. H. Chen, Phys. Rev. E 83, 051915 (2011).

${ }^{19}$ Q. H. Cheng, B. Chen, H. J. Gao, and Y. W. Zhang, J. R. Soc. Interface 9, 283 (2012).

${ }^{20}$ H. Yao and H. Gao, J. Mech. Phys. Solids 54, 1120 (2006).

${ }^{21}$ T. W. Kim and B. Bhushan, J. R. Soc. Interface 5, 319 (2008).

${ }^{22}$ K. Autumn, A. Dittmore, D. Santos, M. Spenko, and M. Cutkosky, J. Exp. Biol. 209, 3569 (2006). 